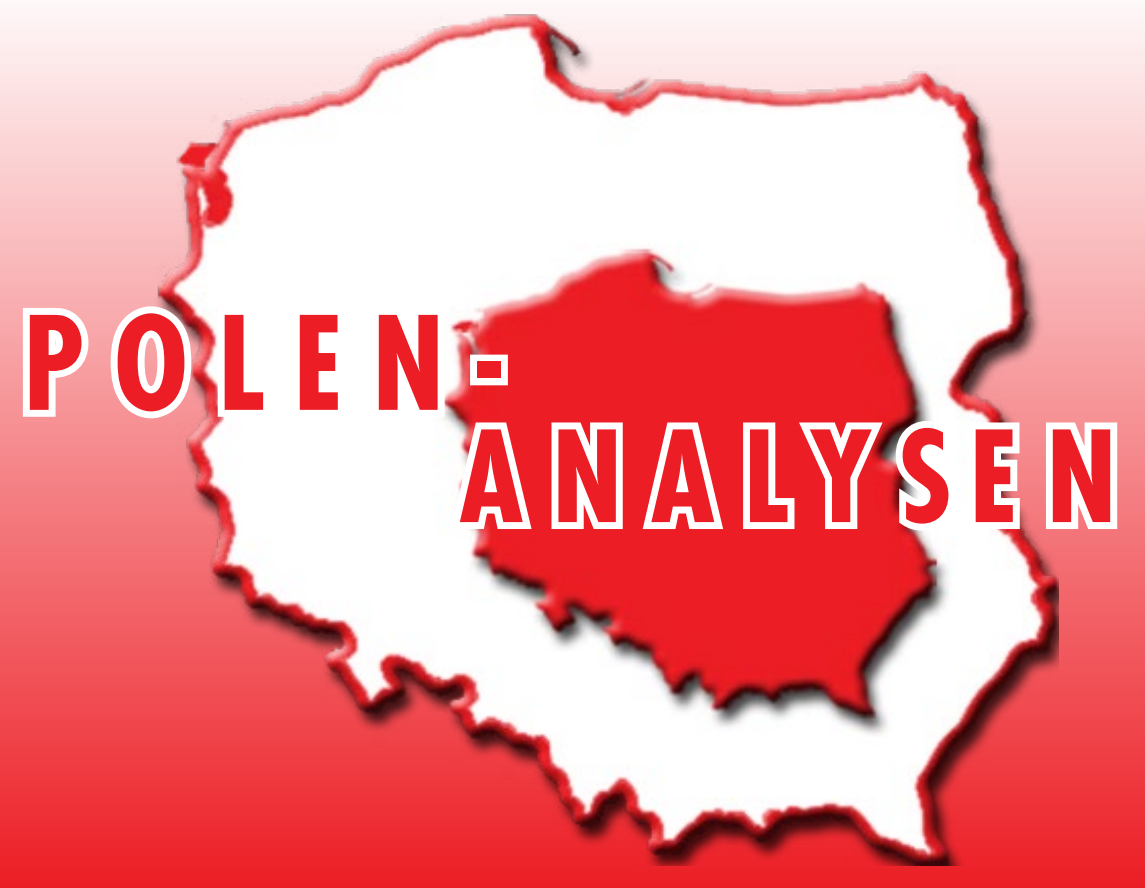

www.laender-analysen.de/polen

\title{
DIE JUNGEN POLEN UND DIE POLITIK
}

ANALYSE

Die jungen Polen und die Politik

Tomasz Szlendak, Thorn

- TABELLEN UND GRAFIKEN ZUM TEXT

Die Ansichten der Wähler zur Lage im Land

\section{CHRONIK}

16. Juni 2015 - 6. Juli 2015

Die nächste Nummer der Polen-Analysen erscheint nach der Sommerpause am Dienstag, den 1. September 2015. Die Redaktion wünscht ihren Leserinnen und Lesern eine erholsame Sommerzeit.

DEUTSCHES POLEN INSTITUT
Forschungsstelle Osteuropa 


\title{
Die jungen Polen und die Politik
}

\author{
Tomasz Szlendak, Thorn
}

\section{Zusammenfassung}

In der Analyse werden drei Thesen entfaltet. Erstens: Die jungen Polen interessieren sich für alles, außer für die institutionalisierte Politik. Zweitens: Die jungen Polen, die an Wahlen teilnehmen, sind sehr dafür, dass sich die politische Bühne verändert, gleichgültig, wer sich solche Veränderungen auf die Fahnen schreibt. Drittens: Die Beteiligung junger Polen an der Politik ist stark emotional geprägt, sie ist wechselhaft, "wolkig« und drückt sich eher in Flashmobs aus als in der institutionalisierten Politik.

$\mathrm{D}$ Politik und die Politiker brauchen ähnlich wie der polnische Staat für die Mehrheit der jungen Polen nicht zu existieren, denn »Staat« bedeutet für die jungen Polen heute eher Facebook, ihre persönlichen Bekannten, ihr Privatleben, ihre persönlichen Leidenschaften und ihre freie Kultur. Für sie sind die staatlichen Strukturen überflüssig, in denen sich nur die Politiker und die Staatsangestellten wohl fühlen, die mit dem System gleichgesetzt werden, mit der schwierigen Lage und der unverständlichen bürokratischen Wand, an die die jungen Menschen stoßen. Die Politiker garantieren ihnen heutzutage nichts und es sieht so aus, als ob sie ihnen nie jemals etwas garantieren werden. Weder eine Ausbildung noch eine Arbeitsstelle noch ein anständiges Gehalt, weder Status noch Sicherheit noch Stabilität, weder eine Wohnung noch eine Rente. So fühlen die jungen Polen. Die Jugend sieht keinen Zusammenhang zwischen ihrer bei Wahlen abgegebenen Stimme und der Wirklichkeit - dahin gehend, dass sich nach den Wahlen etwas tut, insbesondere etwas Gutes für die Jugend.

Diese Gründe sind es in erster Linie, die zu einem konsequenten Desinteresse von fast der Hälfte der jungen Polen an der Politik und den Politikern führen und dazu, dass sie Wahlen wie das Feuer meiden. Fast der Hälfte der Polen und Polinnen sind Wahlen gleichgültig. Im Jahr 2007 gingen 44 Prozent der 18- bis 24-Jährigen nicht zur Wahl sowie 47 Prozent der 25- bis 35-Jährigen. Im Jahr 2008 gaben 45 Prozent der 18- bis 24-Jährigen und 47 Prozent der 25 bis 35-Jährigen keine Stimme ab, im Jahr 2009 entsprechend 52 bzw. 53 Prozent, 2010 46 bzw. 45 Prozent und im Jahr 201148 Prozent der 18- bis 24-Jährigen und 47 Prozent der 25- bis 35-Jährigen. Diese seit Jahren anhaltende Tendenz gilt es sich bewusst zu machen: Fast die Hälfte der jungen Menschen in Polen interessiert sich überhaupt nicht für die Beteiligung an der institutionalisierten Politik.

Schaut man auf diese Abneigung aus einer etwas anderen Datenperspektive, zeigt sich, dass mit 55 Prozent über die Hälfte der Polen im Alter von 18 bis 34 Jahren nicht zur letzten Parlamentswahl (2011) gegangen ist. Warum? Weil das nicht ihr Gelände ist, nicht ihre Spielwiese: Das ist die Arena für irgendwelche unverständ- lichen Spiele und Albernheiten. Die jungen Menschen, die von Soziologen angehalten werden, ihre Abneigung gegenüber dem Staat und der Politik zu begründen, drücken es so aus: Wir werden nicht für Zappelmänner stimmen, weil uns Zirkusauftritte nicht interessieren. Uns interessieren völlig andere Dinge, auf anderen Feldern als im politischen Theater. Wenn die Soziologen die Motivationen der jungen Menschen betrachten, nicht an den Wahlen oder allgemeiner am politischen Leben teilzunehmen, sehen sie, dass sich diese ablehnende Haltung aus der Wahrnehmung der Politik als Clownsarena ergibt. Hinzu kommt, dass der Clown nicht nur ein Possenreißer ist, das heißt eine Figur, die nicht ernstzunehmen ist, sondern auch eine Horrorfigur. Ein Staat mit Gesichtern von Donald Tusk, Jarosław Kaczyński, Janusz Palikot oder Bronisław Komorowski ist für die Jugend ein medialer Horror, eine Arena der Monster, die sich gegenseitig verschlingen. Das, was man als junger Pole oder Polin machen kann, ist, aus der so wahrgenommenen Politik zu desertieren, in die eigenen Welten zu fliehen und sich mit sich selbst zu beschäftigen.

Außerdem sind die institutionalisierten demokratischen Wahlen, der Akt des Wählens mit einer Wahlkommission, für die Jugend ein seltsamer, archaischer Bereich, der außerhalb des Internets liegt, in dem sich ihr soziales Leben abspielt. Wahlen - das ist die alte Welt der etablierten Institutionen, die für die jungen Menschen eine exotische Insel ist. Sie funktionieren in dieser Welt nicht. Sie reiben sich mit ihr gerade in Situationen, wenn jemanden gewählt werden soll, wie bei den letzten Präsidentenwahlen im Mai 2015. Sie gehen dann beispielsweise in einen Kindergarten, wo ein hölzerner weiß-roter Kasten in der Mitte steht, und in diesem Kindergarten soll man etwas mit einem Kugelschreiber auf einem gestempelten Formular ankreuzen und einwerfen. Für junge Menschen wirkt so ein Wahlvorgang mit einer entsprechenden Wahlkommission wie ein Sketch von Monty Python. In der Welt der Jugend wählt man alles und jeden mit Hilfe des Smartphones, man teilt sich direkt und im Alltag in sozialen Netzwerken mit und setzt sein "gefällt mir«. Das ist die Demokratie der jungen Menschen. Die traditionelle Politik ist für sie ein 
Medienzirkus mit Hampelmännern, die man mit den Möglichkeiten des Internets verspotten kann.

In Polen versucht der Staat, sich dauerhaft mit der Nation zu verbinden. Wenn vom Staat gesprochen wird, wird Nation gedacht. Unterdessen geht aus den neuesten Untersuchungen zur Kultur in der Stadt und in der Provinz in Polen hervor, dass es unter den acht Millionen in Polen lebenden jungen Menschen bis zum Alter von 29 Jahren keinen kohärenten kulturellen Kanon gibt, aus dem der Staat eine gemeinsame nationale Identität gestalten könnte, und sei sie auch weit gefasst als Menge gemeinsamer Medien-, Technologie- und BildungsErfahrungen, gemeinsamer "historischer" Erfahrungen, als Regeln des Savoir-vivre, als gemeinsame Sprache und Lebensziele. Die jungen Polen sind im Grunde ein Archipel von Sozio-Inselchen, auf denen separate Leben geführt werden, mit eigenem Stil im Bereich der Kleidung, des privaten und öffentlichen Verhaltens, des Kommunizierens, des Flirtens und der Mobilität. Die jungen Menschen finden natürlich immer andere Menschen, die ihnen nahe sind, aber sie finden sie dank des Internets und seiner Möglichkeiten der sozialen Netzwerke, in denen die gleiche Sprache gesprochen wird, die sie sprechen. Das, was öffentlich ist, kapselt sich in immer mehr und kleinere lifestyle-Nischen $a b$, und die Grenzen dieser Nischen sind gleichzeitig für die jungen Menschen die Grenzen ihrer Welt. Die alten politischen Trennungen, zum Beispiel der auf der politischen Bühne ausgetragene Konflikt zwischen Recht und Gerechtigkeit (Prawo i Sprawiedliwość - PiS) und der Bürgerplattform (Platforma Obywatelska-PO), sind für die Jugend unverständlich, sie berühren nicht die Einteilungen in ihrer Welt, sie befinden sich außerhalb ihrer Welt. Und wenn sie außerhalb ihrer Welt liegen, wenn sie keinen Einfluss auf ihre Welt haben, wenn sich das Leben der Jugend woanders abspielt, dann wird die Politik für die jungen Menschen zu einem Lebensbereich, für den sie sich nicht zu interessieren brauchen.

\section{Veränderung um der Veränderung willen und die ziehende Wolke: die letzten Präsidentenwahlen}

Die, die zurzeit regieren, sind ganz sicher nicht die Lieblinge der jungen Polen. Heute ist eine Generation von Wählern herangereift, die die alten politischen Lager für vollkommen unwesentlich hält. Diejenigen jungen Polen, die zur Wahl gehen, wollen vor allem Veränderungen. Irgendwelche Veränderungen, Hauptsache, es ändert sich etwas auf der betonfesten politischen Bühne, die gegenwärtig von der sogenannten Generation der Solidarność (das heißt der ehemaligen Oppositionellen, die heute zwischen 50 und 75 Jahre alt sind) besetzt wird. Die jungen Polen heute wissen nicht, was die Solidarność überhaupt war, sie wissen nicht, wann der Kriegszustand herrschte und die Volksrepublik Polen assoziieren sie fast ausschließlich mit den Filmkomödien von Stanisław Bareja aus jener Zeit - und mit nichts anderem. Alles, was außerhalb der gegenwärtigen politischen Bühne liegt, zumindest scheint es so, ist attraktiv und wird von der Jugend gewählt, ganz egal, welche Ansichten die neuen Kandidaten vertreten, seien es die Ein-Mandats-Wahlkreise wie Paweł Kukiz oder ultraliberale und zusätzlich misogyne Ansichten wie Janusz Korwin-Mikke.

Außer den Minderheiten, die eine ideologische Orientierung haben, beispielsweise die konservativen katholischen Bewegungen, sind die jungen Menschen mit keiner politischen Partei verbunden. Sie sind eine Wählerschaft, die als eine Wolke beschrieben werden kann, die von einer Partei zur anderen zieht, von der extremen Linken zur extremen Rechten, abhängig von der Situation und den aktuell zur Wahl stehenden Kandidaten. Die jungen Menschen sind also launische Wähler; ihre Wahlentscheidungen werden von ihrer stark emotionalen Weltwahrnehmung bestimmt. Und diese ist ebenfalls »bewölkt« - die jungen Menschen sind unzufrieden, auch wenn nicht völlig klar ist, womit. Aus den Daten der Staatlichen Wahlkommission geht hervor, dass im Jahr 2011 23,3 Prozent der jungen Wähler (zumindest nominell und erklärtermaßen) für die liberal-linke Palikot-Bewegung (Ruch Palikota) gestimmt hatten. Das Gesamtwahlergebnis dieser Partei betrug 10 Prozent. Diese Wolke der Unterstützung der Jugend für Palikot zog vier Jahre später bei den Präsidentenwahlen zu Paweł Kukiz und zu Janusz Korwin-Mikke, konservativ-rechten Politikern, die radikal andere Slogans verkündeten als der antiklerikale Palikot. Wesentlich ist hier, dass beide Parteien und die genannten Politiker bei den jungen Wählern Emotionen wecken, radikal sind und die Zerstörung der bisherigen politischen Ordnung verkünden - und dabei ist es vollkommen egal, was und welche konkreten Veränderungen sie meinen. Paweł Kukiz ist die gleiche Eintagsfliege und der gleiche Showman wie damals Palikot. Schlicht und einfach neu, anders und nicht alteingesessen auf der politischen Bühne.

Im ersten Wahlgang der Präsidentenwahlen (10. Mai 2015) unterstützten die jüngsten Wähler Paweł Kukiz und in der Stichwahl (24. Mai 2015) Andrzej Duda. Nach Angaben des Marktforschungsinstituts IPSOS stimmten über 41 Prozent der 18- bis 29-jährigen Wähler für Kukiz (in der Gesamtwählerschaft waren es 20,5 Prozent), 20,7 Prozent für Andrzej Duda, 13,8 Prozent für den Amtsinhaber Bronisław Komorowski und für den radikalen und sehr kontroversen Liberalen Janusz Korwin-Mikke 13,6 Prozent (3,26 Prozent der Gesamt- 
wählerschaft). Es ist also sehr deutlich, dass die Stimmen der jungen Wähler den Kandidaten zuteil werden, die bisher nicht auf der politischen Bühne sichtbar waren - Kukiz und paradoxerweise Duda, der bis dato ein in Polen nicht bemerkter Europaparlamentarier von PiS war -, oder aber solchen Politikern, die von den jungen Menschen als Outsider identifiziert werden wie Korwin-Mikke.

Die Jugend traf ihre Wahl keineswegs aufgrund einer fundierten Unterstützung für irgendeinen dieser Politiker, die den jungen Polen überwiegend vollkommen unbekannt waren. Zwar ist Paweł Kukiz ein 52 Jahre alter Rockstar, aber seine musikalischen Erfolge liegen Jahre zurück und werden eher von den Eltern der heute jüngsten Kukiz-Wähler gefeiert als von diesen selbst. Die Wahl für Kukiz oder Duda ist auch keine Unterstützung einer politischen Option, denn hinter Kukiz steht gar keine Partei und bisher hat er auch keine eigene gegründet, und PiS, die Duda aufgestellt hat, wird von der Mehrheit der jungen Polen und Polinnen als archaisch verurteilt. PiS betrachten die jungen Menschen als "zukunftsunfähige« Partei, die ihnen nichts Neues offeriert und von Ressentiments und Abrechnungen mit den politischen Feinden lebt. Allerdings war sie bei den letzten Präsidentenwahlen im Stande, ihr "zukunftsunfähiges" Gesicht, konkret Jarosław Kaczyński oder Antoni Macierewicz, hinter dem Gesicht des mit nichts identifizierten Andrzej Duda zu verbergen. Im ersten Wahlgang der Präsidentenwahlen stimmte für Andrzej Duda ein ähnlicher Prozentsatz junger Wähler wie vier Jahre zuvor bei den Parlamentswahlen für PiS gestimmt hatte (20,7 bzw. 23,8 Prozent). Betrachtet man die Unterstützung für PiS unter den jungen Menschen im Jahresdurchschnitt, war sie dreimal geringer als die Unterstützung in dieser Altersgruppe für die $P O$ und oszillierte zwischen 2007 und 2011 zwischen 8 und 13 Prozent in der Gruppe der 18- bis 24-Jährigen und zwischen 8 und 12 Prozent in der Gruppe der 25- bis 35-Jährigen. Die $P O$ verlor ihren großen Vorsprung vor $P i S$ in den letzten Präsidentenwahlen deutlich, als sich diese hinter Andrzej Duda zurückzog, der nicht mit ihr assoziiert wurde. In der Stichwahl zogen die jungen Wähler ihre Stimme von Kukiz oder Korwin-Mikke ab und gaben sie Duda, um nur nicht Komorowski zu wählen, der für Stillstand, die alte Welt und die alte politische Bühne steht.

Dieses Ergebnis in dieser Wählergruppe ist eindeutig Ausdruck von Protest. Die zahlreichen Subwelten, in denen die jungen Menschen heute leben, und die Jugend-Mikrokulturen haben keine politische Repräsentation. Oder anders ausgedrückt: Auf die hybriden Bedürfnisse und Identitäten der Jugend antwortet keine der großen Parteien in besonderer Weise. Paweł Kukiz mit seiner hybriden Identität - ein bisschen Rockstar, Volkstribun, Showman, eine Type im T-Shirt und emotional werdender Aktivist - ist den jungen Menschen ein bisschen ähnlich. Er ist ihnen noch am nächsten und am besten verdaulich. Vor allem aber war er gegen alles, was auch ihnen, vor allem aus kulturell-ästhetischen Gründen, nicht gefällt.

Nicht zutreffend ist meiner Meinung nach die These, dass die für Kukiz abgegebenen Stimmen der jungen Wähler eine Art Auflehnung gegen ihre schlechte wirtschaftliche Lage seien, gegen ihre befristeten Verträge und miserable Bezahlung. De facto hat die Mehrheit der jungen Menschen, die zur Wahl gehen, insbesondere diejenigen in den Städten, dank der intergenerationellen Transfers etwas, wovon sie bis in die 30er leben kann. Zum großen Teil sind dies "Nesthocker«, die vollständig oder teilweise von ihren Eltern unterhalten werden, aber nicht Menschen, die Hunger leiden. Zwar sind sie die gesellschaftliche Gruppe, die über das niedrigste Einkommen verfügt, aber hier geht es nicht ums Geld. Wenn überhaupt, geht es um die institutionell-strukturelle Wand, die ihrer Meinung nach vor acht Jahren von der Bürgerplattform aufgebaut wurde. Hinzu kommt die Kombination aus ästhetischem Widerwillen und Langeweile. Die jungen Menschen haben ganz einfach genug von der seit knapp einem Jahrzehnt, also im Grunde so lange sie sich erinnern, regierenden Bürgerplattform. Sie haben genug von der offen zur Schau getragenen Tendenz, sich mit sich selbst zu befassen, eine Haltung, die als Faulheit und komplette Abwendung vom Leben unten identifiziert wird. Staatspräsident Bronisław Komorowski war für sie die Repräsentation des Establishment, das so unwahrscheinlich weit von ihren Lebensstilen entfernt ist und so archaisch in seinen Aussagen und Handlungen, dass er es wert war, ihm ein »Nein!« entgegenzusetzen.

Merkwürdig ist das insofern, als im Ergebnis der Präsidentenwahlen der katholische neokonservative Liberale durch den katholischen, neoliberalen Konservativen ersetzt wurde. Ich habe keinen Zweifel daran, dass dies aus der Perspektive eines großen Teils der jungen Wähler dieselben Personen sind. Sehr gefallen haben mir die Bezeichnungen, die nach dem Wahlkampf, unmittelbar vor den Wahlen auf Twitter die Runde machten. Es hieß dort, es würde Bigos mit Pudding kämpfen. Komorowski wurde nach dem traditionellen polnischen Kohlgericht Bigos genannt, weil er altmodisch und traditionell ist, wie ein polnischer Adliger aus einer anderen Zeit. Duda wurde dagegen Pudding getauft, weil er die Konsistenz von Pudding hat, er ist weich und gestaltlos. Diese Metaphern geben meiner Ansicht nach hervorragend wieder, was die jungen Menschen über die Wahl zwischen den beiden Kandidaten dachten. 
Wie schon gesagt, sind die jungen Menschen in Polen stark zersplittert, verpackt in ihre kleinen Subwelten mit unterschiedlichen kulturellen Kanons, Verhaltensregeln und Werten. Bildlich gesprochen suchen die einen eifrig nach einem Restaurant mit italienischen Speisen, andere mit französischer cuisine, wieder andere eine Sushibar oder eine location mit veganen Burgern - aber keiner von ihnen sucht ein Restaurant, in dem Bigos und Pudding serviert werden. Die polnische politische Bühne ist ein Restaurant, in dem Speisen angeboten werden, auf die die jungen Menschen absolut keine Lust haben. Aber sie müssen etwas essen. Das ist der Zwang der Demokratie. Von zwei Übeln war der Twitter-Pudding noch das kleinere. Die Stimme für Andrzej Duda war ein Protest gegen das ganze politische Restaurant mit seiner vorgestrigen, überhaupt nicht auf die Gäste abgestimmten Speisekarte. Hinzu kam, dass »Bigos" Komorowski schon zu lange serviert worden war. Es ist schlicht und einfach das Flehen der Jugend um Personalveränderungen auf der politischen Bühne. Es ging der Jugend darum, gegen Komorowski zu stimmen. Es reichte aus, die Kommunikation im polnischen Internet zu verfolgen, die vor der Stichwahl immer grausamer wurde. Hier wurde der noch amtierende Präsident als alter polnischer Bär dargestellt, der von einem Fettnäpfchen ins andere tappt. Ein Bär, der in einem vorzeitlichen Wald sitzt, abgeschnitten von der heutigen polnischen Stadt und dem polnischen jungen Alltag. Die letzten zwei Wochen Wahlkampf machten Komorowski völlig zunichte. Er gelangte überhaupt nicht in die Kommunikationskanäle, die von jungen Menschen unter 30 genutzt werden. Für diese war er nur in den Ausdrucksformen des Internets sichtbar, deren Urheber sie selbst waren. Die Jugend in Polen ist demnach gegen alles, was alt ist oder alt zu sein scheint. $P O$ und $P i S$ sind für sie weitestgehend ein und dasselbe. POPiS - alte Männer, die die polnische politische Wirklichkeit verwalten, so lange die Erinnerungen reichen, die nicht imstande sind, ihnen etwas zu geben, die ihnen aber viel nehmen.

Der gewählte Präsident, Andrzej Duda, ist dabei gleichfalls archaisch, traditionell und katholisch, was es schwer macht, die Entscheidung der Jugend zu verstehen. Jedoch hat meiner Ansicht nach Dudas Katholizismus keine besondere Bedeutung, denn die jungen Polen sind zunehmend weltlich eingestellt und in Fragen der Lebensgestaltung sind sie mehrheitlich weit von den Einflüssen der Religion entfernt. Die Wahl Andrzej Dudas war kein ideelles Bekenntnis. Ob der Kandidat sagt, dass Gott wichtig sei oder nicht, hat für die Jugend deshalb keine besondere Bedeutung, weil so eine Erklärung nicht unmittelbar ihr Privatleben berührt, ihre Altersgruppe oder die Facebook-Instagram-Struktur ihre Alltags. Auch wenn in Polen nun nach den Präsidenten- wahlen oder nach den Parlamentswahlen im Herbst eine stärkere Reorientierung der politischen Bühne in Richtung »Religiosität« eintreten würde, wenn angefangen würde, politische Botschaften mit religiöser Symbolik zu verbinden, würden es die jungen Menschen nicht einmal bemerken. Fragen der Lebensgestaltung sind in ihrem Leben wesentlich, aber die Regierenden haben schließlich keine wirksamen Kontrollinstrumente oder Zwangsmaßnahmen, um bestimmte Verhaltensregeln in diesem Bereich durchzusetzen. Duda war schlicht und einfach ein neues Gesicht; er war nicht Komorowski.

Die "junge-Wähler-Wolke» kann sich deshalb frei von links nach rechts bewegen, weil die Jugend hybrid ist, was ihre Werte angeht. In ihren Identitäten lassen sich beispielsweise locker Homophobie und Anarchismus verbinden. Ich kann eine junge Polin sein, die nicht in religiösen Kategorien denkt und linke Ansichten vertritt, und gleichzeitig Janusz Korwin-Mikke oder Andrzej Duda wählen, weil ich gerade in der Laune bin, dem, was ist, einen Tritt zu geben und meine Wut zu zeigen. Die Jugend ist eine unvorhersehbare Wählerschaft für die Parteispezialisten, die sich mit politischem Marketing beschäftigen, denn ihre Ansichten sind nicht auf die alte Weise »abgepackt «. Die traditionelle Verbindung von linken Ansichten über den Staat und liberaler Lebensgestaltung muss hier nicht notwendig bestehen. Ihre Ansichten sind hybrid und ein für die Spezialisten für politisches Marketing völlig unverständlicher ephemerer Nebel, der von Partei zu Partei zieht und von Kandidat zu Kandidat und keinen dauerhaften politischen Präferenzen zugeschrieben oder angeklebt werden kann, denn solche Präferenzen gibt es ganz einfach nicht. Weil es diese stabilen Präferenzen nicht gibt, stimmen die jungen Menschen in Polen auch zum Spaß für kontroverse Kandidaten oder sie geben ungültige Stimmen ab, weil ihnen keiner der Kandidaten passt.

\section{Die politische Verfasstheit der Jugend außerhalb der Politik}

Die politische Verfasstheit der jungen Polen und Polinnen kommt nicht in den institutionalisierten demokratischen Wahlen zum Ausdruck und wird dies auch in Zukunft nicht. Wenn die Jugend heutzutage einen Aufruhr organisiert, dann findet er andernorts statt, so wie der Protest gegen das internationale Abkommen ACTA im Jahr 2011. Diese Empörung zeugt davon, dass in den jungen Menschen ein großes Potential für Aufruhr steckt. Natürlich können sich die jungen Menschen ernähren, aber sie stoßen an das Unvermögen, die eigenen Bedürfnisse zu verwirklichen, an die kulturelle und strukturelle Wand. Das ganze Leben lang, in der Schule, in der Familie, in den Medien wurden sie auf die eigene Außergewöhnlichkeit abgerichtet. Sie werden 
die ganze Zeit von ihren Telefonen und Tablets angestarrt. Sie sind auf sich selbst bezogen, auto-obsessiv, und der Arbeitsmarkt in Polen bietet ihnen eine miserable Bezahlung und dienende Funktionen an. Plötzlich, im Zusammenstoß mit diesem Markt, hören sie auf, außergewöhnlich zu sein. Dieser Zusammenprall mit der polnischen Erwachsenenwelt, die nicht in der Lage ist, ihre besonderen Eigenschaften und Kompetenzen aufzunehmen und zu nutzen, mündet zurzeit in Aktionen der De-Individuation, das heißt gemeinschaftlichen Ausbrüchen wie der Protest gegen ACTA.

Wir haben es in Polen mit einer Ellenbogengesellschaft zu tun, mit einer Gesellschaft von sehr individuellen Einzelwesen, die auf sich selbst fixiert sind. Das beginnt schon in der polnischen Schule, die sich konkreten Einzelwesen und ihren Leistungen widmet und diese mit denen der anderen vergleicht. Der Jugend fehlen Momente der Gemeinschaft, die nur bei solchen Ereignissen wie beispielsweise den Protestmärschen als Reaktion auf Gewaltverbrechen auftreten. Dann gehen sie gemeinsam, getragen von Emotionen. Dies ist eine Art gesellschaftlicher Trance, gemeinschaftlicher, momentaner Obsession, man versetzt sich in soziale Schwingungen: Wir hören auf, wir selbst zu sein, wir werden endlich eine Gruppe. Der Protest gegen ACTA war so etwas. Er war eher ein Karneval der gemeinschaftlichen Trance als eine politische Aktion. Andere Beispiele sind die Märsche für die Unabhängigkeit oder der Protest der Palikot-Anhänger gegen die Verteidiger des Kreuzes auf der Krakowskie Przedmieście-Straße in Warschau. Bei den jungen Menschen ist die Sehnsucht nach Gemeinschaft sichtbar, aber Gemeinschaft wird nirgendwo in Polen aufgebaut. Die jungen Menschen können sich nur in solchen entindividualisierten Aktionen verwirklichen. Manche jungen Polen, die mit einem geringen kulturellen Kapital ausgestattet sind, leben sich in der Hooliganbewegung aus - weil das heftig ist, weil es gegen etwas ist und weil es ein Gefühl von Gemeinschaft gibt. Ich muss nicht überlegen, welche Idee dahinter steht, was meine Identität ist. Ich verschmelze mit der Gruppe. Diese Energie konzentriert sich nicht um eine Idee, sondern um Ereignisse.

Ich denke, dass die Jugend mit ihren Ausbrüchen sozialer Wut nicht die rhythmisierten politischen Rituale wie Wahlen steuern wird, sondern nur Skandale, plötzliche Ereignisse, die sie als gegen sich gerichtet auffasst. Ähnlich wie beim Aufruhr gegen ACTA können beispielsweise radikale Polizeiaktionen gegen den Besitz von Marihuana tranceartige Wutausbrüche hervorrufen oder wenn den jungen Wählern das Subjekt abgesprochen oder ihre Kompetenzen in Frage gestellt werden, wenn sie in Kategorien altersbedingter Dummheit beschrieben werden, als Kinder, die nichts anderes machen, als auf ihren Smartphones herumzutippen. Auf diese Weise wurden sie von Repräsentanten der Erwachsenenwelt, zum Beispiel dem einflussreichen Chefredakteur der Tageszeitung»Gazeta Wyborcza», Adam Michnik, nach den letzten Präsidentenwahlen charakterisiert.

Übersetzung aus dem Polnischen: Silke Plate

\section{Über den Autor}

Prof. Dr. Tomasz Szlendak (Jahrgang 1974) ist Direktor des Instituts für Soziologie an der Nikolaus Kopernikus Universität in Thorn (Uniwersytet Mikołaja Kopernika w Toruniu). Im Rahmen der Familien- und Jugendsoziologie sowie der Konsumsoziologie und Evolutionspsychologie liegen seine Forschungsschwerpunkte auf den Entwicklungen der Kultur und des Lebensstils des Westens. Er schreibt und kommentiert für meinungsbildende Tageszeitungen und Magazine wie Gazeta Wyborzca, Polityka und Kultura Liberalna. 


\section{Die Ansichten der Wähler zur Lage im Land}

Grafik 1: Die Wähler von Paweł Kukiz nach Altersgruppen der Gesamtwählerschaft

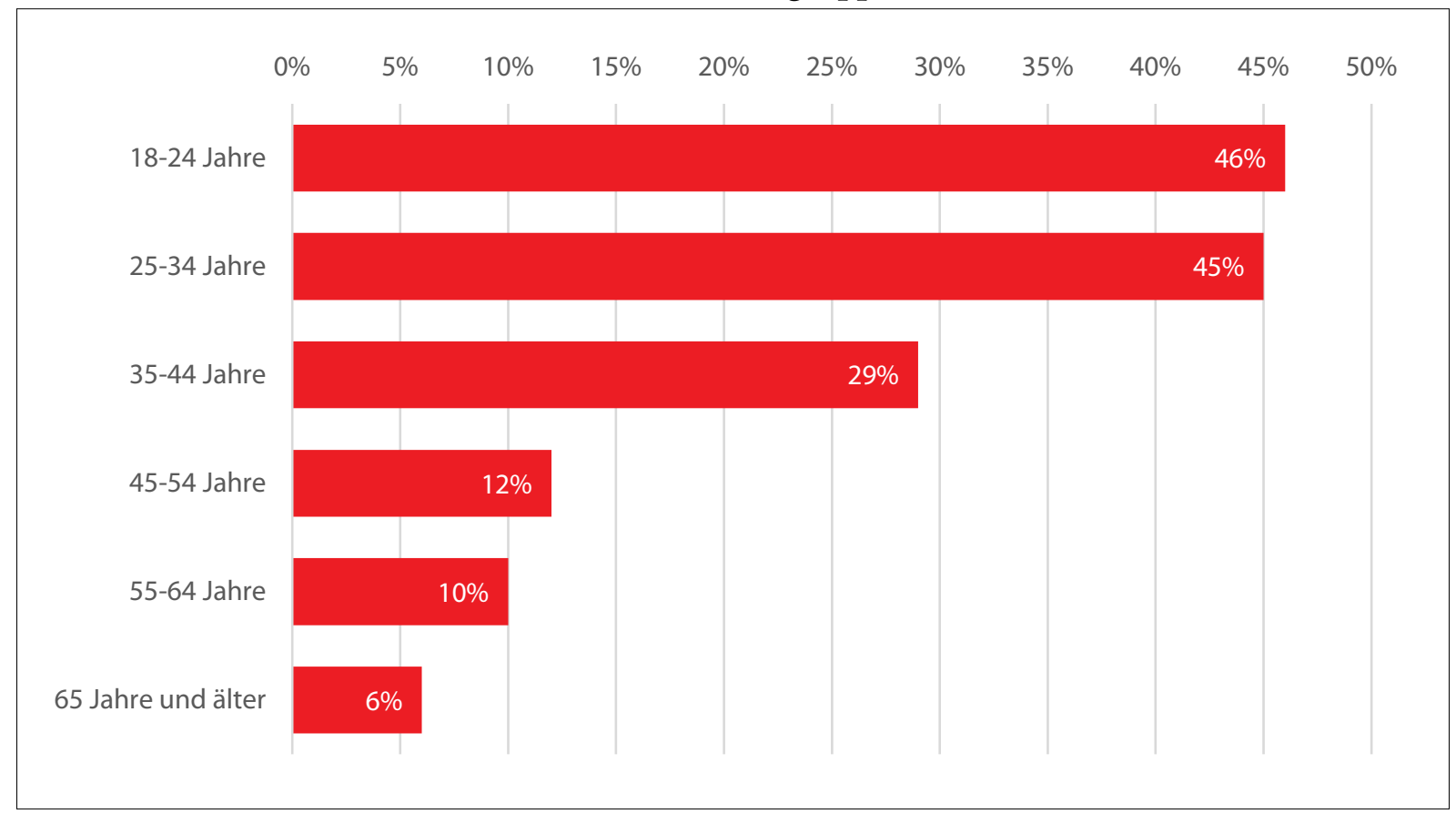

Anm.: Die Befragten gaben an, im ersten Wahlgang der Präsidentenwablen 2015 Pawet Kukiz gewählt zu haben.

Quelle: CBOS Nr 86/2015: Kim są wyborcy Pawła Kukiza? [Wer sind die Wähler von Paweł Kukiz?] Warszawa 06/2015. <www.cbos.pl>

Grafik 2: Die Wähler von Paweł Kukiz nach deklarierten politischen Ansichten

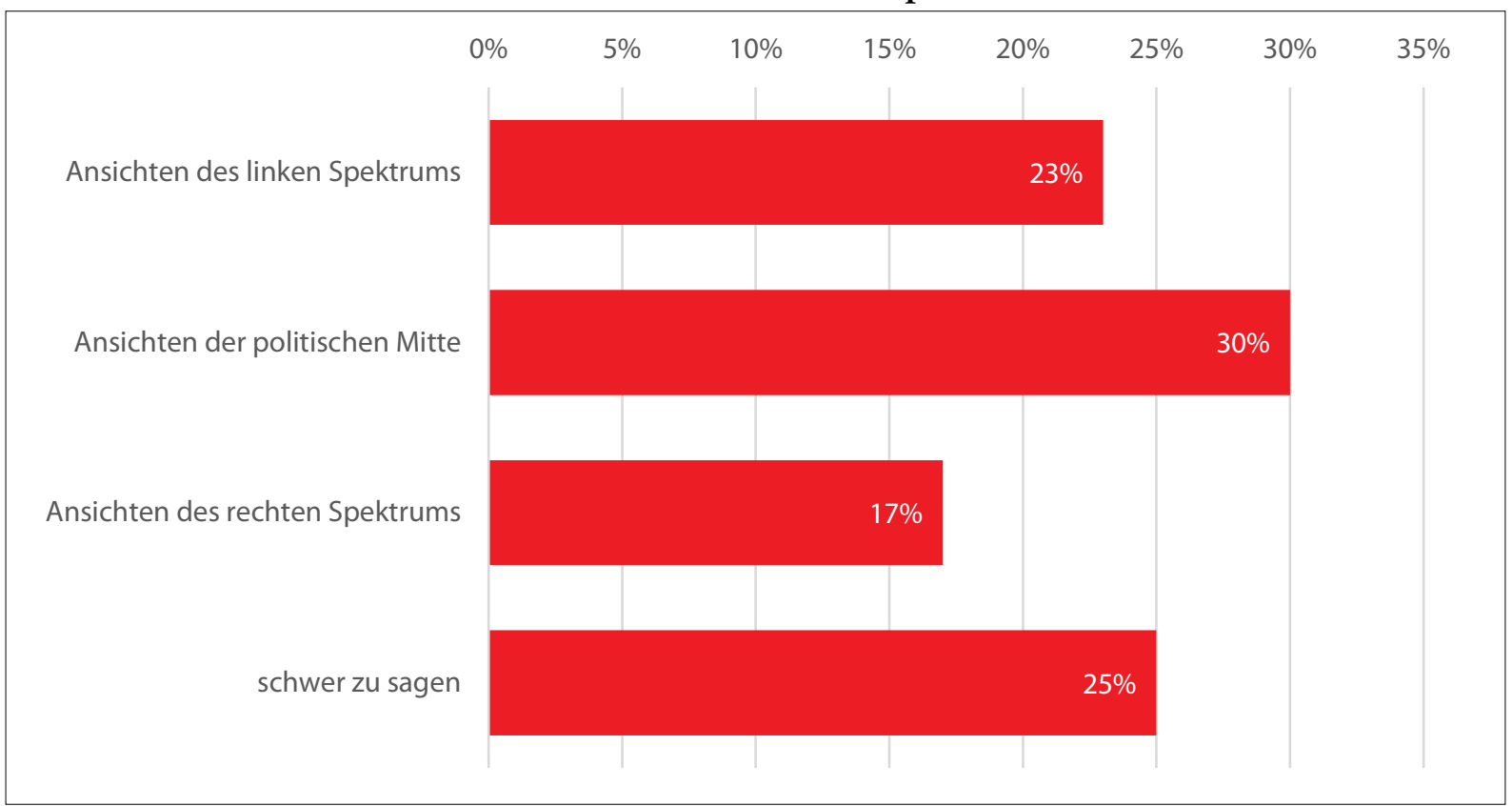

Anm.: Die Befragten gaben an, im ersten Wablgang der Präsidentenwablen 2015 Pawet Kukiz gewählt zu haben.

Quelle: CBOS Nr 86/2015: Kim sa wyborcy Pawła Kukiza? [Wer sind die Wählervon Pawet Kukiz?] Warszawa 06/2015. <www.cbos.pl> 
Grafik 3: Läuft die Situation in Polen im Allgemeinen in eine gute oder in eine schlechte Richtung? Antworten derer, die angaben, im ersten Wahlgang der Präsidentenwahlen 2015 ... gewählt zu haben

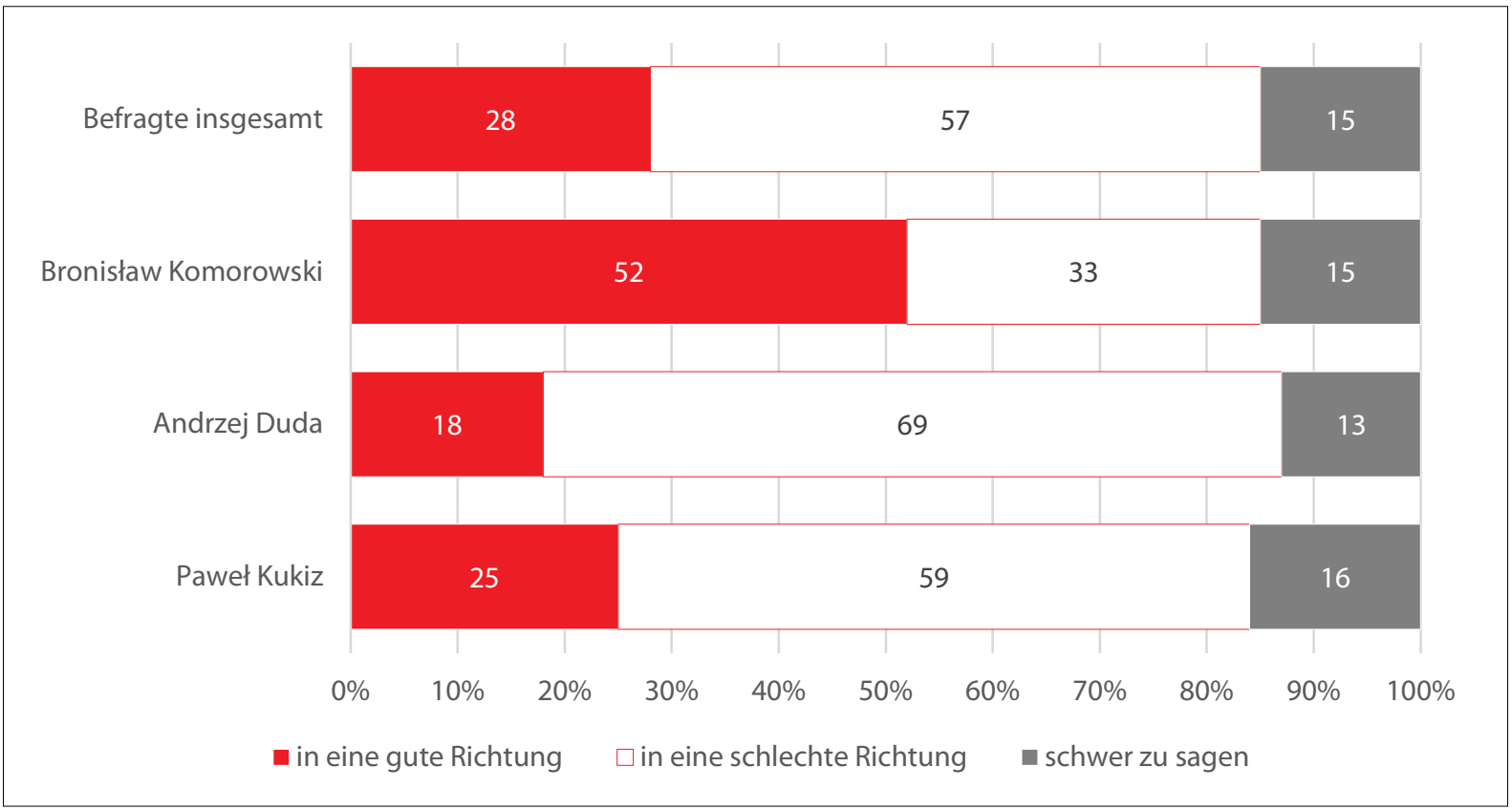

Quelle: CBOS Nr 86/2015: Kim sa wyborcy Pawła Kukiza? [Wer sind die Wähler von Pawet Kukiz?] Warszawa 06/2015. <www.cbos.pl>

Grafik 4: Wie beurteilen Sie insgesamt die aktuelle politische Situation in Polen? Antworten derer, die angaben, im ersten Wahlgang der Präsidentenwahlen 2015 ... gewählt zu haben

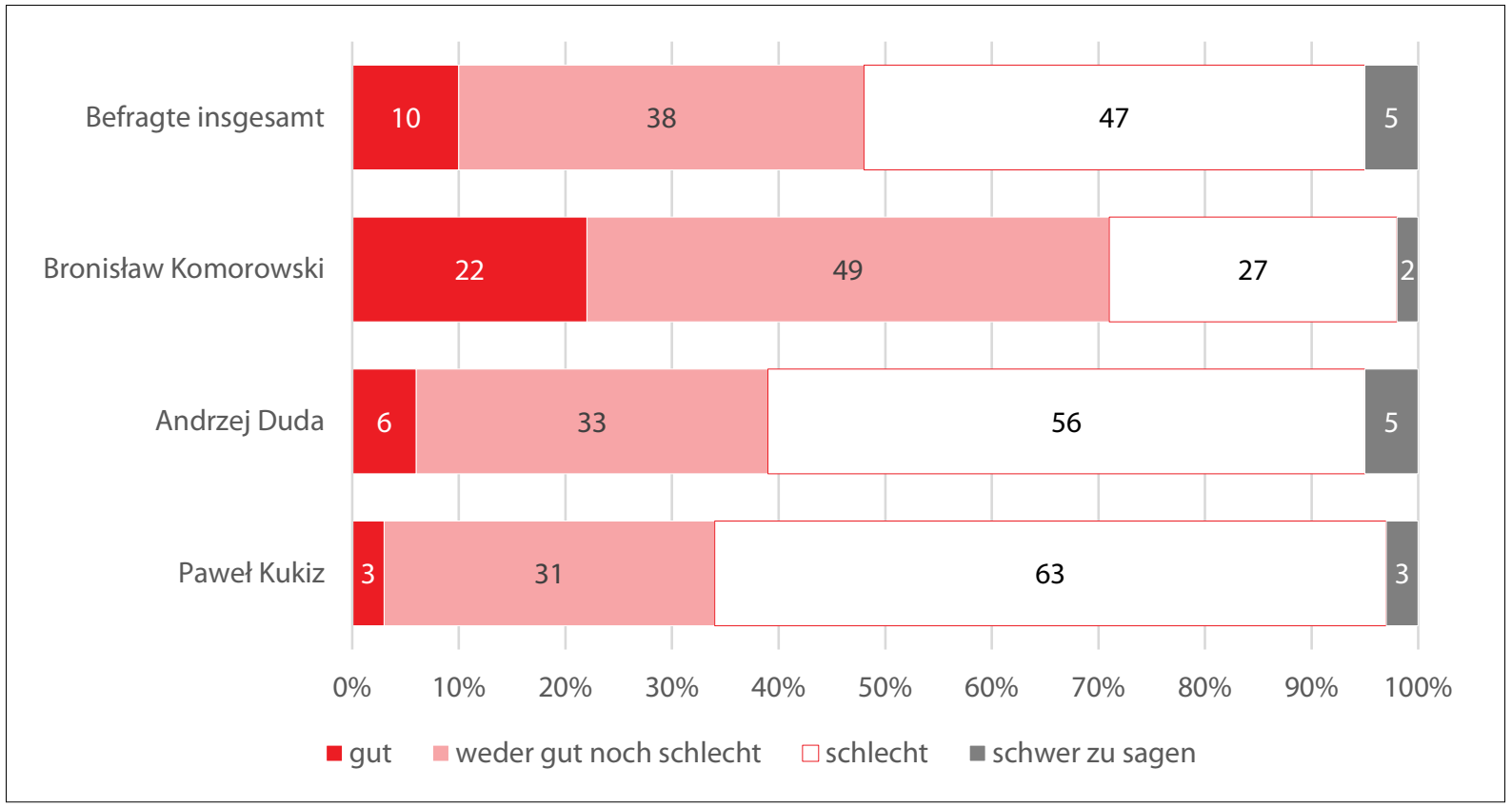

Quelle: CBOS Nr 86/2015: Kim sq wyborcy Pawła Kukiza? [Wer sind die Wähler von Pawet Kukiz?] Warszawa 06/2015. <www.cbos.pl> 
Grafik 5: Wie beurteilen Sie insgesamt die aktuelle wirtschaftliche Situation in Polen? Antworten derer, die angaben, im ersten Wahlgang der Präsidentenwahlen 2015 ... gewählt zu haben

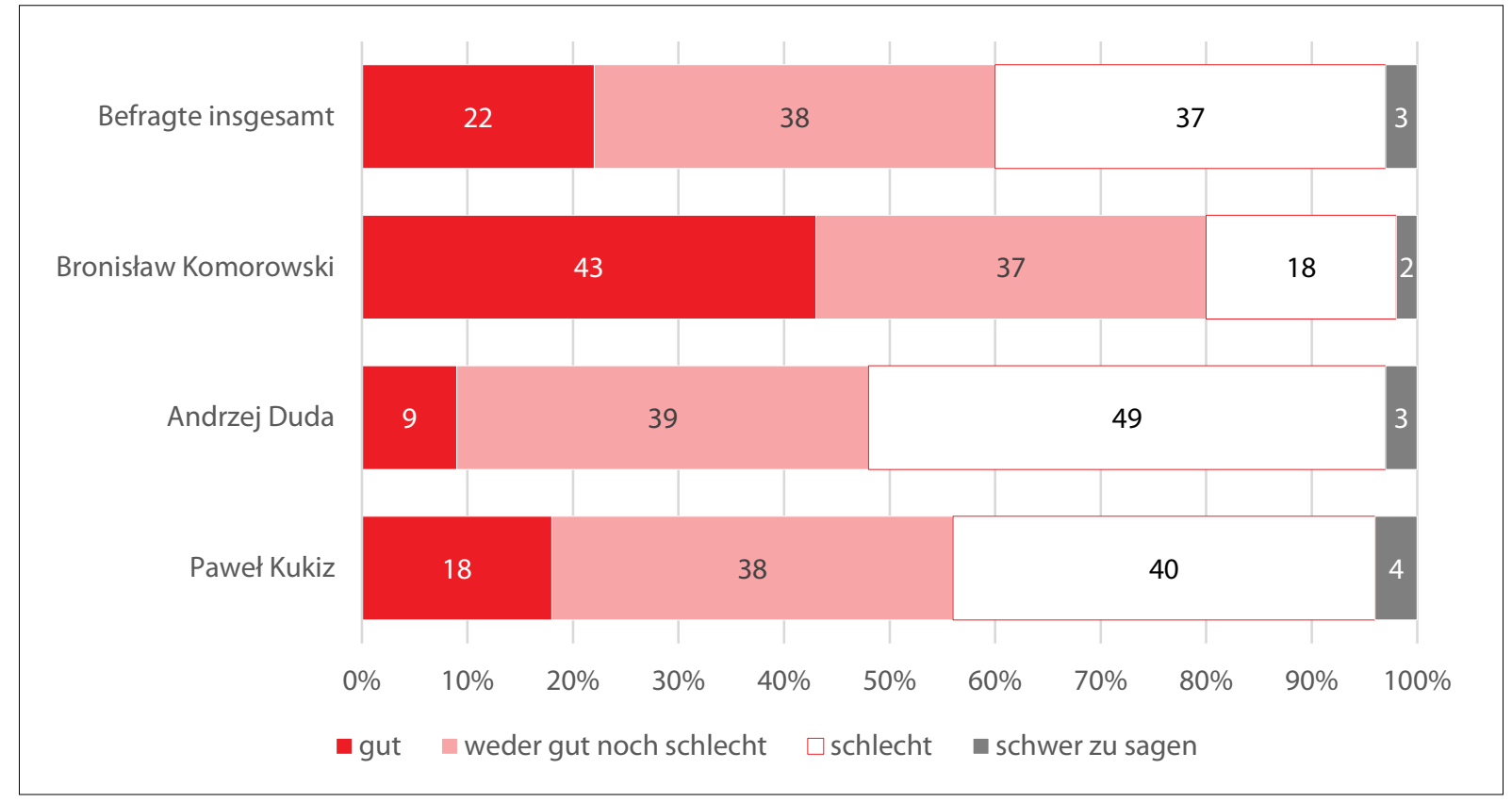

Quelle: CBOS Nr 86/2015: Kim sq wyborcy Pawła Kukiza?. [Wer sind die Wähler von Pawet Kukiz?] Warszawa 06/2015. <www.cbos.pl>

Grafik 6: Wird sich im Laufe des kommenden Jahres die Situation in Polen verbessern, nicht verändern oder verschlechtern? Antworten derer, die angaben, im ersten Wahlgang der Präsidentenwahlen 2015 ... gewählt zu haben

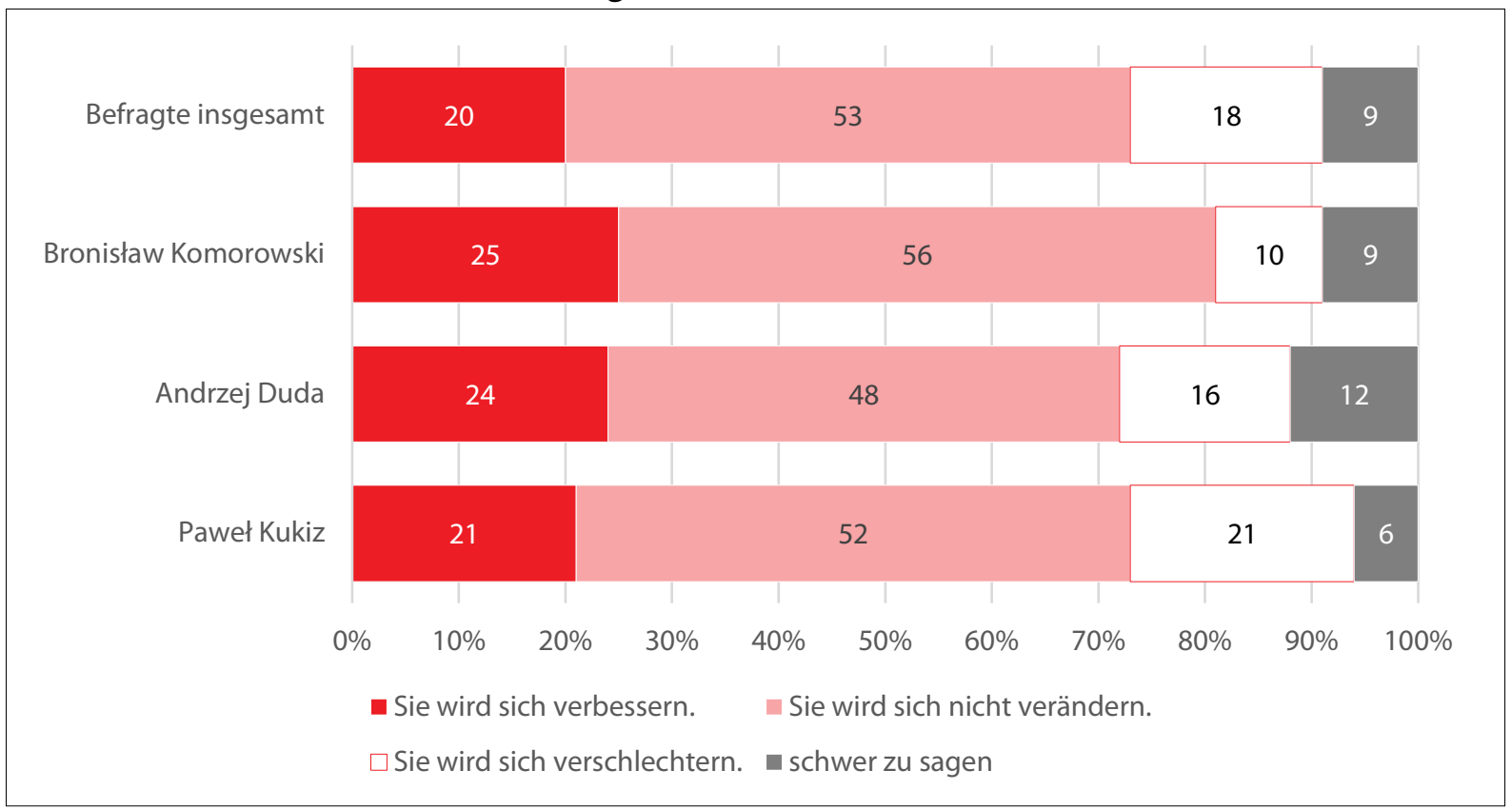

Quelle: CBOS Nr 86/2015: Kim sq wyborcy Pawła Kukiza? [Wer sind die Wähler von Pawet Kukiz?] Warszawa 06/2015. <www.cbos.pl> 
Grafik 7: Sind Sie im Allgemeinen zufrieden oder unzufrieden mit der Art und Weise, wie die Demokratie in Polen funktioniert? Antworten derer, die angaben, im ersten Wahlgang der Präsidentenwahlen 2015 ... gewählt zu haben

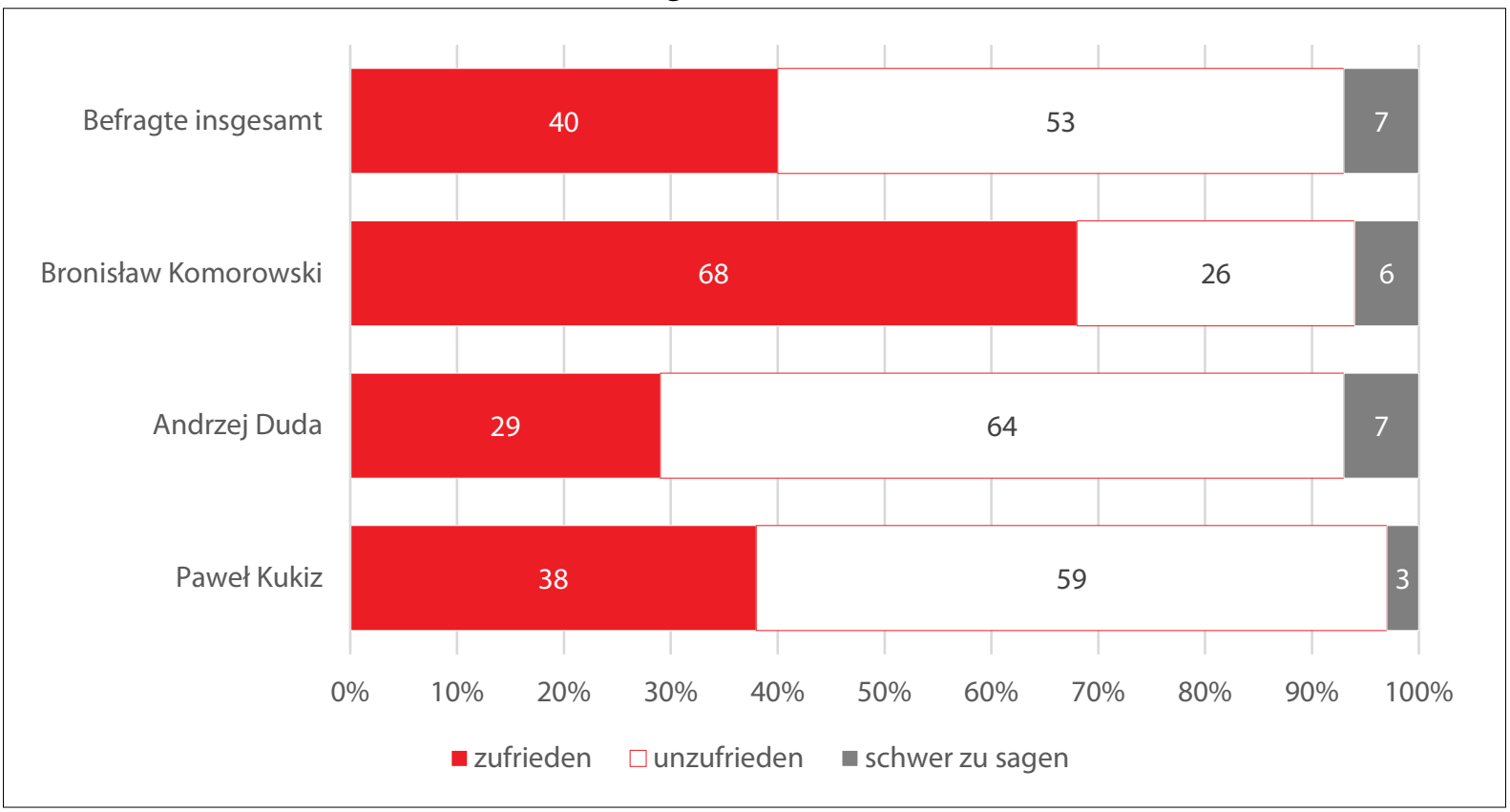

Quelle: CBOS Nr 86/2015: Kim sa wyborcy Pawła Kukiza? [Wer sind die Wähler von Pawet Kukiz?] Warszawa 06/2015. <www.cbos.pl>

Tabelle 1: Ansichten der Wähler zu ausgewählten gesellschaftspolitischen Fragen

\begin{tabular}{|c|c|c|c|c|}
\hline \multirow[t]{2}{*}{$\begin{array}{l}\text { Angaben auf } \\
\text { einer pro-contra-Skala } \\
\text { von } 1 \text { bis } 7\end{array}$} & \multicolumn{3}{|c|}{$\begin{array}{c}\text { Zustimmung der Befragten, die angaben, im ersten Wahl- } \\
\text { gang der Präsidentenwahlen } 2015 \ldots \text {.... gewählt zu haben } \\
(\%)\end{array}$} & \multirow[t]{2}{*}{$\begin{array}{l}\text { Befragte } \\
\text { insgesamt } \\
\quad(\%)\end{array}$} \\
\hline & Paweł Kukiz & Andrzej Duda & $\begin{array}{l}\text { Bronisław } \\
\text { Komorowski }\end{array}$ & \\
\hline $\begin{array}{l}\text { Polen sollte nach möglichst } \\
\text { enger Integration im Rahmen } \\
\text { der EU streben. }(1+2+3)\end{array}$ & 30 & 23 & 46 & 33 \\
\hline ambivalente Meinung (4) & 25 & 13 & 20 & 21 \\
\hline $\begin{array}{l}\text { Polen sollte nach möglichst } \\
\text { weit reichender Unabhängig- } \\
\text { keit in der EU streben. } \\
(5+6+7)\end{array}$ & 44 & 59 & 31 & 41 \\
\hline schwer zu sagen & 1 & 5 & 3 & 5 \\
\hline
\end{tabular}


Tabelle 1: Ansichten der Wähler zu ausgewählten gesellschaftspolitischen Fragen (Fortsetzung)

\begin{tabular}{|c|c|c|c|c|}
\hline \multirow[t]{2}{*}{$\begin{array}{l}\text { Angaben auf } \\
\text { einer pro-contra-Skala } \\
\text { von } 1 \text { bis } 7\end{array}$} & \multicolumn{3}{|c|}{$\begin{array}{c}\text { Zustimmung der Befragten, die angaben, im ersten Wahl- } \\
\text { gang der Präsidentenwahlen } 2015 \ldots . . \text { gewählt zu haben } \\
(\%)\end{array}$} & \multirow[t]{2}{*}{$\begin{array}{l}\text { Befragte } \\
\text { insgesamt } \\
\quad(\%)\end{array}$} \\
\hline & Paweł Kukiz & Andrzej Duda & $\begin{array}{c}\text { Bronisław } \\
\text { Komorowski }\end{array}$ & \\
\hline $\begin{array}{l}\text { Der Staat sollte den Bürgern } \\
\text { ein hohes Sozialleistungs- } \\
\text { niveau garantieren wie } \\
\text { Gesundheitsfürsorge, Schul- } \\
\text { wesen usw. }(1+2+3)\end{array}$ & 75 & 88 & 77 & 83 \\
\hline ambivalente Meinung (4) & 10 & 9 & 13 & 8 \\
\hline $\begin{array}{l}\text { Die Bürger sollten für ihre } \\
\text { Gesundheitsfürsorge, die } \\
\text { Möglichkeiten der Ausbildung } \\
\text { ihrer Kinder etc. selbst sorgen. } \\
(5+6+7)\end{array}$ & 15 & 3 & 10 & 8 \\
\hline schwer zu sagen & 0 & 0 & 0 & 1 \\
\hline $\begin{array}{l}\text { Diejenigen, die gut verdienen, } \\
\text { sollten eine höhere Einkom- } \\
\text { mensteuer zahlen als die, die } \\
\text { wenig verdienen. }(1+2+3)\end{array}$ & 60 & 76 & 64 & 64 \\
\hline ambivalente Meinung (4) & 11 & 6 & 11 & 9 \\
\hline $\begin{array}{l}\text { Alle sollten unabhängig von } \\
\text { der Höhe ihres Einkommens } \\
\text { denselben Einkommensteuer- } \\
\text { satz zahlen. }(5+6+7)\end{array}$ & 28 & 14 & 25 & 24 \\
\hline schwer zu sagen & 1 & 4 & 0 & 3 \\
\hline $\begin{array}{l}\text { Straftaten müssen hart be- } \\
\text { straft werden, auch wenn dies } \\
\text { die Rechte und Freiheiten der } \\
\text { durchschnittlichen Bürger } \\
\text { einschränken sollte. }(1+2+3)\end{array}$ & 31 & 44 & 38 & 42 \\
\hline ambivalente Meinung (4) & 11 & 11 & 17 & 12 \\
\hline $\begin{array}{l}\text { Straftaten müssen bekämpft } \\
\text { werden, aber so, dass die } \\
\text { Rechte und Freiheiten der } \\
\text { durchschnittlichen Bürger } \\
\text { nicht eingeschränkt werden. } \\
(5+6+7)\end{array}$ & 56 & 41 & 45 & 44 \\
\hline schwer zu sagen & 2 & 4 & 0 & 2 \\
\hline $\begin{array}{l}\text { Der Schwangerschaftsabbruch } \\
\text { sollte ohne Einschränkungen } \\
\text { erlaubt sein. }(1+2+3)\end{array}$ & 38 & 11 & 39 & 28 \\
\hline ambivalente Meinung (4) & 32 & 25 & 32 & 28 \\
\hline $\begin{array}{l}\text { Der Schwangerschaftsabbruch } \\
\text { sollte vollkommen verboten } \\
\text { sein. }(5+6+7)\end{array}$ & 28 & 57 & 26 & 39 \\
\hline schwer zu sagen & 2 & 7 & 3 & 5 \\
\hline
\end{tabular}


Tabelle 1: Ansichten der Wähler zu ausgewählten gesellschaftspolitischen Fragen (Fortsetzung)

\begin{tabular}{|c|c|c|c|c|}
\hline \multirow[t]{2}{*}{$\begin{array}{l}\text { Angaben auf } \\
\text { einer pro-contra-Skala } \\
\text { von } 1 \text { bis } 7\end{array}$} & \multicolumn{3}{|c|}{$\begin{array}{c}\text { Zustimmung der Befragten, die angaben, im ersten Wahl- } \\
\text { gang der Präsidentenwahlen } 2015 \text {.... gewählt zu haben } \\
(\%)\end{array}$} & \multirow[t]{2}{*}{$\begin{array}{l}\text { Befragte } \\
\text { insgesamt } \\
\quad(\%)\end{array}$} \\
\hline & Paweł Kukiz & Andrzej Duda & $\begin{array}{c}\text { Bronisław } \\
\text { Komorowski }\end{array}$ & \\
\hline $\begin{array}{l}\text { Das Konkordat zwischen } \\
\text { Polen und dem Apostolischen } \\
\text { Stuhl ist nicht notwendig. Der } \\
\text { Staat sollte kein Bekenntnis } \\
\text { und keine Kirche hervor- } \\
\text { heben. }(1+2+3)\end{array}$ & 52 & 24 & 54 & 43 \\
\hline ambivalente Meinung (4) & 16 & 12 & 15 & 14 \\
\hline $\begin{array}{l}\text { Das Konkordat ist notwendig. } \\
\text { Der Staat sollte vor allem } \\
\text { mit der katholischen Kirche } \\
\text { zusammenarbeiten, der die } \\
\text { Mehrheit der Bevölkerung } \\
\text { angehört. }(5+6+7)\end{array}$ & 31 & 61 & 30 & 39 \\
\hline schwer zu sagen & 1 & 3 & 1 & 4 \\
\hline $\begin{array}{l}\text { Die Rechtsprechung sollte } \\
\text { die Möglichkeit der formalen } \\
\text { Partnerschaft zwischen gleich- } \\
\text { geschlechtlichen Personen } \\
\text { zulassen. }(1+2+3)\end{array}$ & 33 & 14 & 44 & 26 \\
\hline ambivalente Meinung (4) & 18 & 5 & 10 & 11 \\
\hline $\begin{array}{l}\text { Die Rechtsprechung sollte } \\
\text { keinesfalls die Möglichkeit } \\
\text { der formalen Partnerschaft } \\
\text { zwischen gleichgeschlecht- } \\
\text { lichen Personen zulassen. } \\
(5+6+7)\end{array}$ & 47 & 78 & 43 & 59 \\
\hline schwer zu sagen & 2 & 3 & 3 & 4 \\
\hline $\begin{array}{l}\text { Polen sollte so schnell wie } \\
\text { möglich den Euro einführen. } \\
(1+2+3)\end{array}$ & 14 & 6 & 19 & 14 \\
\hline ambivalente Meinung (4) & 14 & 10 & 25 & 16 \\
\hline $\begin{array}{l}\text { Polen sollte den Euro über- } \\
\text { haupt nicht einführen. } \\
(5+6+7)\end{array}$ & 66 & 81 & 52 & 66 \\
\hline schwer zu sagen & 6 & 3 & 4 & 4 \\
\hline
\end{tabular}


Tabelle 1: Ansichten der Wähler zu ausgewählten gesellschaftspolitischen Fragen (Fortsetzung)

\begin{tabular}{|c|c|c|c|c|}
\hline \multirow[t]{2}{*}{$\begin{array}{l}\text { Angaben auf } \\
\text { einer pro-contra-Skala } \\
\text { von } 1 \text { bis } 7\end{array}$} & \multicolumn{3}{|c|}{$\begin{array}{c}\text { Zustimmung der Befragten, die angaben, im ersten Wahl- } \\
\text { gang der Präsidentenwahlen } 2015 \ldots . . . \text { gewählt zu haben } \\
(\%)\end{array}$} & \multirow[t]{2}{*}{$\begin{array}{l}\text { Befragte } \\
\text { insgesamt } \\
\quad(\%)\end{array}$} \\
\hline & Paweł Kukiz & Andrzej Duda & $\begin{array}{c}\text { Bronisław } \\
\text { Komorowski }\end{array}$ & \\
\hline $\begin{array}{l}\text { Polen sollte eine aktive Ost- } \\
\text { politik betreiben und die } \\
\text { proeuropäischen Bemühungen } \\
\text { der Ukrainer und anderer } \\
\text { Nationen der ehemaligen } \\
\text { UdSSR unterstützen. }(1+2+3)\end{array}$ & 19 & 23 & 34 & 25 \\
\hline ambivalente Meinung (4) & 36 & 20 & 25 & 24 \\
\hline $\begin{array}{l}\text { Polen sollte sich nicht auf } \\
\text { besondere Weise im Osten } \\
\text { engagieren, sondern sich vor } \\
\text { allem um gute Beziehungen } \\
\text { zu Russland kümmern. } \\
(5+6+7)\end{array}$ & 43 & 50 & 37 & 44 \\
\hline schwer zu sagen & 2 & 7 & 4 & 7 \\
\hline $\begin{array}{l}\text { Am wichtigsten sind die } \\
\text { Beschäftigungsgarantie und } \\
\text { der Schutz der existierenden } \\
\text { Arbeitsplätze, sogar wenn sich } \\
\text { die wirtschaftliche Situation } \\
\text { verschlechtert. }(1+2+3)\end{array}$ & 39 & 45 & 25 & 40 \\
\hline ambivalente Meinung (4) & 20 & 16 & 22 & 19 \\
\hline $\begin{array}{l}\text { Das wichtigste ist, dass die } \\
\text { Unternehmen schnell auf } \\
\text { Veränderungen der wirt- } \\
\text { schaftlichen Situation } \\
\text { reagieren können, damit sie } \\
\text { bei Problemen auf einfache } \\
\text { Weise die Beschäftigung } \\
\text { reduzieren können und sich } \\
\text { trauen, erneut Arbeitnehmer } \\
\text { einzustellen. }(5+6+7)\end{array}$ & 38 & 29 & 49 & 34 \\
\hline schwer zu sagen & 3 & 10 & 4 & 7 \\
\hline $\begin{array}{l}\text { Alle staatlichen Unternehmen } \\
\text { sollen privatisiert werden. } \\
(1+2+3)\end{array}$ & 23 & 7 & 19 & 13 \\
\hline ambivalente Meinung (4) & 18 & 10 & 18 & 15 \\
\hline $\begin{array}{l}\text { Eine deutliche Anzahl von } \\
\text { Unternehmen sollte in staatli- } \\
\text { chem Besitz bleiben. }(5+6+7)\end{array}$ & 59 & 80 & 62 & 67 \\
\hline schwer zu sagen & 0 & 3 & 1 & 5 \\
\hline
\end{tabular}




\section{Juni 2015 - 6. Juli 2015}

\begin{tabular}{|c|c|}
\hline 16.06 .2015 & $\begin{array}{l}\text { Staatspräsident Bronisław Komorowski vereidigt die neuen Minister für Gesundheit, Sport und den Staats- } \\
\text { schatz, Marian Zembala, Adam Korol und Andrzej Czerwiński. Vorausgegangen waren die Rücktritte der bis- } \\
\text { herigen Ressortchefs im Zusammenhang mit der "Abhöraffäre". }\end{array}$ \\
\hline 16.06 .2015 & $\begin{array}{l}\text { Im Europäischen Parlament entsteht unter dem Vorsitz von Marine Le Pen (Front National) die Fraktion Europa } \\
\text { der Nationen und der Freiheit. Ihr gehören zwei polnische Abgeordnete, Michał Marusik und Stanisław Żółtek, } \\
\text { vom Kongress der Neuen Rechten (Kongres Nowej Prawicy - KNP) an. }\end{array}$ \\
\hline 17.06 .2015 & $\begin{array}{l}\text { Der neu vereidigte Schatzminister Andrzej Czerwiński nennt als eine der wichtigsten Aufgaben in seiner Amts- } \\
\text { zeit die Fertigstellung des Flüssiggasterminals in Swinemünde (Ŝwinoujście). }\end{array}$ \\
\hline 18.06 .2015 & $\begin{array}{l}\text { Staatspräsident Bronisław Komorowski empfängt in Warschau NATO-Generalsekretär Jens Stoltenberg. } \\
\text { Komorowski bekräftigt die Erwartungen Polens, dass die rotierende Anwesenheit von NATO-Truppen in } \\
\text { Polen in eine ständige Stationierung von NATO-Einheiten umgewandelt wird. }\end{array}$ \\
\hline 19.06 .2015 & $\begin{array}{l}\text { Ministerpräsidentin Ewa Kopacz und Verteidigungsminister Tomasz Siemoniak nehmen am Treffen der Vise- } \\
\text { grád-Gruppe (Polen, Slowakei, Tschechien, Ungarn) in Bratislava (Slowakei) teil. Zur Flüchtlingspolitik der } \\
\text { Europäischen Union sagt Kopacz, dass sich Polen solidarisch bei der Aufnahme von Flüchtlingen zeigen werde. } \\
\text { Es müssten aber alle Entscheidungen über konkrete Maßnahmen in der Kompetenz des betreffenden EU-Lan- } \\
\text { des liegen und freiwillig sein. }\end{array}$ \\
\hline 20.06.2015 & $\begin{array}{l}\text { Auf dem Parteikonvent von Recht und Gerechtigkeit (Prawo i Sprawiedliwość - PiS) in Warschau spricht sich } \\
\text { Parteivorsitzender Jarosław Kaczyński für Beata Szydło als Kandidatin für das Amt der Ministerpräsidentin } \\
\text { aus. Die Parlamentswahlen werden im Herbst stattfinden. }\end{array}$ \\
\hline 21.06 .2015 & $\begin{array}{l}\text { Infolge eines Hackerangriffs auf das Computersystem der polnischen Fluggesellschaft LOT werden } 20 \text { Flüge } \\
\text { abgesagt; zahlreiche Flüge verspäten sich. Zirka } 1.400 \text { Passagiere sind betroffen. }\end{array}$ \\
\hline 22.06 .2015 & $\begin{array}{l}\text { Arbeitsminister Władysław Kosiniak-Kamysz (Polnische Bauernpartei/Polskie Stronnictwo Ludowe-PSL) teilt } \\
\text { mit, dass seine Fraktion den Gesetzentwurf zum Rentenbeginn nach } 40 \text { Beitragsjahren im Sejm eingereicht } \\
\text { hat. Die PSL hatte den Vorschlag von Staatspräsident Bronisław Komorowski übernommen, der den Entwurf } \\
\text { nach seiner Niederlage bei der Wahl für eine zweite Amtszeit zurückgezogen hatte, da er keine wesentlichen } \\
\text { Entscheidungen bis zum Ende seiner Amtszeit mehr zu treffen gedenkt. }\end{array}$ \\
\hline 23.06 .2015 & $\begin{array}{l}\text { Im Sejm wird des 50. Jahrestages des Briefes der polnischen Bischöfe an ihre deutschen Amtsbrüder während } \\
\text { des II. Vatikanischen Konzils gedacht, in dem die Bischöfe die wegweisende Formulierung "Wir vergeben und } \\
\text { bitten um Vergebung« verwendeten. Der Brief wird im Rahmen einer Ausstellung im Sejm zur Versöhnung zwi- } \\
\text { schen Polen und Deutschland nach dem Zweiten Weltkrieg erstmalig im Original gezeigt. }\end{array}$ \\
\hline 24.06 .2015 & $\begin{array}{l}\text { Die Regierungsbeauftragte für Sicherheitsfragen, Urszula Augustyn, stellt im Sejm das Regierungsprogramm für } \\
\text { das Schulwesen 2015-2018 "Sicher+« vor. Es umfasst die Bereiche Internet, Umgang mit gefährlichen Situatio- } \\
\text { nen und Öffnung der Schulen für nicht-schulische Freizeitorganisationen. Das Programm löst das Vorgänger- } \\
\text { programm »Sichere und freundliche Schule« ab und stellt } 75 \text { Mio. Zloty zur Verfügung. }\end{array}$ \\
\hline 25.06 .2015 & $\begin{array}{l}\text { Die Kandidatin der Regierungskoalition, Małgorzata Kidawa-Błońska (Bürgerplattform/Platforma Obywatel- } \\
\text { ska-PO), wird vom Sejm mit } 244 \text { von insgesamt } 441 \text { Stimmen zur neuen Sejmmarschallin gewählt. Für ihren } \\
\text { Gegenkandidaten Jerzy Wenderlich (Demokratische Linksallianz/Sojusz Lewicy Demokratycznej-SLD) stim- } \\
\text { men } 43 \text { Abgeordnete. Hintergrund ist der Rücktritt von Sejmmarschall Radosław Sikorski im Zuge neuer Ent- } \\
\text { wicklungen in der "Abhöraffäre«. Kidawa-Błońska war bisher Regierungssprecherin. }\end{array}$ \\
\hline 26.06 .2015 & $\begin{array}{l}\text { Das Umweltministerium gibt bekannt, dass 11,6 Mio. Zloty für lokale Initiativen zum Schutz der Umwelt und } \\
\text { für eine nachhaltige Entwicklung bereitgestellt werden. }\end{array}$ \\
\hline 27.06.2015 & $\begin{array}{l}\text { Beata Szydło, Kandidatin von Recht und Gerechtigkeit (Prawo i Sprawiedliwość - PiS) für das Amt der Minis- } \\
\text { terpräsidentin, spricht sich dafür aus, beim Referendum am 6. September zusätzlich über die Senkung des Ren- } \\
\text { teneintrittsalters, die Schulpflicht für Sechsjährige und die Privatisierung der polnischen Waldgebiete entschei- } \\
\text { den zu lassen. Die von Staatspräsident Bronisław Komorowski anberaumte Volksbefragung beinhaltet Entschei- } \\
\text { dungen über das Wahlrecht, die Parteienfinanzierung und das Steuerrecht. }\end{array}$ \\
\hline 29.06 .2015 & $\begin{array}{l}\text { Der ehemalige Vorsitzende der Demokratischen Linksallianz (Sojusz Lewicy Demokratycznej - SLD) Grzegorz } \\
\text { Napieralski und der unabhängige Parlamentsabgeordnete Andrzej Rozenek geben die Gründung der neuen Par- } \\
\text { tei „Die Weiß-Roten« (Biało-Czerwoni) bekannt. Napieralski, der am Wochenende seinen Austritt aus der SLD } \\
\text { mitgeteilt hat, zeigt sich an einer möglichen Zusammenarbeit mit seiner ehemaligen Partei nicht interessiert, da } \\
\text { Parteichef Miller eher ausschließe als integrierend handele. }\end{array}$ \\
\hline
\end{tabular}




\begin{tabular}{|c|c|}
\hline 30.06 .2015 & $\begin{array}{l}\text { Das Kabinett von Ministerpräsidentin Ewa Kopacz tagt in Kattowitz (Katowice) und berät das Programm } \\
\text { „Schlesien } 2.0 \text { - Ein Programm zur Unterstützung der Woiwodschaft Schlesien und des Westlichen Kleinpo- } \\
\text { len«. Dieses war in Folge der Bergarbeiterproteste Anfang des Jahres entwickelt worden und umfasst Investiti- } \\
\text { onsmaßnahmen und Umstrukturierungen im Bergbau. Kopacz betont, dass sich die polnische Energiesicher- } \\
\text { heit auf die Kohleenergie stützt. }\end{array}$ \\
\hline 01.07 .2015 & $\begin{array}{l}\text { Der Rat für Nationale Sicherheit (Rada Bezpieczeństwa Narodowego - RBN) berät die Vorbereitungen des } \\
\text { NATO-Gipfels im Juli } 2016 \text { in Warschau. Auf der Pressekonferenz im Anschluss unterstreicht Staatspräsident } \\
\text { Bronisław Komorowski, dass Polen darauf Einfluss nehmen werde, dass nicht nur die rotierende Anwesenheit } \\
\text { von Truppen des Bündnisses im östlichen Europa aufrechterhalten wird, sondern auch die ständige Anwesenheit } \\
\text { der NATO verstärkt wird. Dazu gehören auch Waffenmagazine, die eine schnelle Vorbereitung eines eventuel- } \\
\text { len Verteidigungsfalls ermöglichen. Weiter werde Polen bei den Bündnispartnern für die Erhöhung der natio- } \\
\text { nalen Verteidigungshaushalte auf zwei Prozent des Bruttoinlandsprodukts werben. }\end{array}$ \\
\hline 02.07 .2015 & $\begin{array}{l}\text { Das Finanzministerium teilt mit, dass zwischen Polen und China ein Memorandum zur Vereinbarung des Infor- } \\
\text { mationsaustauschs im Falle von Geldwäsche und Terrorismusfinanzierung unterzeichnet worden ist. Ähnliche } \\
\text { Vereinbarungen bestehen bereits mit } 82 \text { Ländern. }\end{array}$ \\
\hline 03.07 .2015 & $\begin{array}{l}\text { Ministerpräsidentin Ewa Kopacz und ihr slowakischer Amtskollege Robert Fico unterzeichnen in Siemanowice } \\
\text { Śląskie eine Absichtserklärung über Zusammenarbeit im Rüstungsbereich. Kopacz teilt mit, dass die Slowakei } \\
\text { in den nächsten drei Jahren } 30 \text { gepanzerte Fahrzeuge des Typs Rosomak von Polen kaufen wird. }\end{array}$ \\
\hline 04.07 .2015 & $\begin{array}{l}\text { Auf dem dreitägigen Parteitag des vereinigten rechten Spektrums in Kattowitz (Katowice) nehmen außer Recht } \\
\text { und Gerechtigkeit (Prawo i Sprawiedliwość - PiS) auch Polen Gemeinsam (Polska Razem) und Solidarisches } \\
\text { Polen (Solidarna Polska) teil. Beata Szydło, Kandidatin für das Amt der Ministerpräsidentin, stellt das Partei- } \\
\text { programm vor, dessen Schwerpunkte auf der Arbeits- und Sozialpolitik liegen. }\end{array}$ \\
\hline 06.07 .2015 & $\begin{array}{l}\text { Nach aktuellen Angaben des Innenministeriums wurden im Jahr } 2014170.000 \mathrm{~m}^{2} \text { Wohnraum in Polen an } \\
\text { Ausländer verkauft; an erster Stelle an Deutsche mit } 18 \% \text { der Gesamtfläche, gefolgt von Ukrainern mit } 14 \% \text {. }\end{array}$ \\
\hline
\end{tabular}

Sie können die gesamte Chronik seit 2007 auch auf <http://www.laender-analysen.de/polen/> unter dem Link "Chronik« lesen. 
Die Polen-Analysen erscheinen zweimal monatlich als E-Mail-Dienst. Sie werden gemeinsam vom Deutschen PolenInstitut Darmstadt, der Bremer Forschungsstelle Osteuropa und der Deutschen Gesellschaft für Osteuropakunde herausgegeben.

Ein Archiv der Polen-Analysen finden Sie im Internet unter <www.laender-analysen.de/polen>

Kostenloses Abonnement unter <http://www.deutsches-polen-institut.de/Newsletter/subscribe.php>

Diese Analysen finden Sie online als Lizenzausgabe auf

$<$ bpb.de>

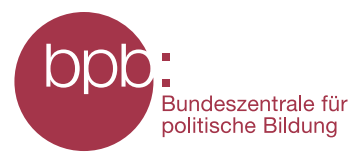

\section{Deutsches Polen-Institut Darmstadt}

Das Deutsche Polen-Institut Darmstadt (DPI) ist ein Forschungs-, Informations-, und Veranstaltungszentrum für polnische Kultur, Geschichte, Politik, Gesellschaft und die deutsch-polnischen Beziehungen, die sich im Kontext der europäischen Integration entwickeln. Das seit März 1980 aktive und bis 1997 von Gründungsdirektor Karl Dedecius geleitete Institut ist eine Gemeinschaftsgründung der Stadt Darmstadt, der Länder Hessen und Rheinland-Pfalz sowie des Bundes. 1987 wurden die Kultusminister der Länder und 2011 das Auswärtige Amt weitere institutionelle Träger. Einen wesentlichen Beitrag zur Verwirklichung der Institutsziele leisten private Stiftungen. Das DPI hat satzungsgemäß die Aufgabe, durch seine Arbeit zur Vertiefung der gegenseitigen Kenntnisse des kulturellen, geistigen und gesellschaftlichen Lebens von Polen und Deutschen beizutragen.

Ziel der Vermittlertätigkeit des DPI ist es, »die zu interessieren, auf die es politisch, wirtschaftlich, gesellschaftlich und kulturell im deutsch-polnischen Verhältnis ankommt (Leitlinien 1997). Es geht um die Entscheider und Multiplikatoren in Politik, Kultur, Bildung, Verwaltung, Medien und Wirtschaft und, wesentlich stärker ausgeprägt als bisher, um das Hineinwirken in Wissenschaft, Forschung und Bildung.

Derzeit bemüht sich das DPI in Kooperation mit den verstreuten Orten wissenschaftlicher Polen-Kompetenz an deutschen Hochschulen und Forschungsinstituten verstärkt darum, ausgehend von einer Bestandsaufnahme deutscher Polen-Forschung Ort wissenschaftlicher Forschung und verbindendes, vernetzendes und kooperierendes Zentrum zu werden. Ausgangspunkt der Neuausrichtung ist die kaum mehr kontrollierbare Dynamik des Rückbaus der Ressourcen der wissenschaftlichen Polen-Kompetenz in den unterschiedlichen Disziplinen. Mit der über 68.000 Bände zählenden multidisziplinären Fachbibliothek für Polen, die eine einzigartige Sammlung polnischer Literatur in der Originalsprache und in deutscher Übersetzung umfasst, ist das DPI bereits ein geschätzter Ort der Recherche und des wissenschaftlichen Arbeitens. (<www.deutsches-polen-institut.de $>$ )

Forschungsstelle Osteuropa an der Universität Bremen (<www.forschungsstelle.uni-bremen.de $>$ ) 1982 gegründet, widmet sich die Forschungsstelle Osteuropa an der Universität Bremen der interdisziplinären Analyse der Länder Ost- und Ostmitteleuropas in Zeitgeschichte und Gegenwart. Der Forschungsschwerpunkt liegt dabei auf der Rolle von "Dissens und Konsens«, von Opposition und Zivilgesellschaft in ihrem historischen, politischen, gesellschaftlichen und kulturellen Kontext. Die Forschungsstelle besitzt in ihrem Archiv eine einzigartige Sammlung alternativer Kulturgüter und unabhängiger Texte aus den ehemaligen sozialistischen Ländern. Darunter befindet sich auch eine umfangreiche Sammlung des "Zweiten Umlaufs«, die das Schrifttum und Dokumente unabhängiger Initiativen und gesellschaftlicher Gruppen in Polen aus der Zeit von 1976 bis zum Umbruch umfasst. Hinzu kommt eine umfangreiche Bibliothek mit wissenschaftlicher Literatur. Mit Archiv, Bibliothek und zwei wissenschaftlichen Abteilungen ist die Forschungsstelle auch eine Anlaufstelle sowohl für Gastwissenschaftler als auch für die interessierte Öffentlichkeit.

Eine der Hauptaufgaben der Forschungsstelle ist die Information der interessierten Öffentlichkeit. Dazu gehören unter anderem regelmäßige E-Mail-Informationsdienste für Politik, Wirtschaft, Zivilgesellschaft und Medien.

Die Meinungen, die in den Polen-Analysen geäußert werden, geben ausschließlich die Auffassung der Autoren wieder.

Abdruck und sonstige publizistische Nutzung sind nach Rücksprache mit der Redaktion gestattet.

Redaktion: Prof. Dr. Dieter Bingen (verantwortlich) (Darmstadt), Silke Plate, M.A. (Bremen) Technische Gestaltung: Matthias Neumann

Polen-Analysen-Layout: Cengiz Kibaroglu, Matthias Neumann

Alle Ausgaben der Polen-Analysen sind mit Themen- und Autorenindex archiviert unter www.laender-analysen.de

Die Polen-Analysen werden im Rahmen der Datenbank World Affairs Online (WAO) ausgewertet und sind im Portal IREON www.ireon-portal.de recherchierbar.

ISSN 1863-9712 @ 2015 by Deutsches Polen-Institut Darmstadt und Forschungsstelle Osteuropa, Bremen

Kontakt: Dr. Andrzej Kaluza, Presse- und Öffentlichkeitsarbeit, Deutsches Polen-Institut, Mathildenhöhweg 2,

D-64287 Darmstadt, Tel.: 06151/4985-13, Fax: 06151/4985-10, E-Mail: polen-analysen@dpi-da.de, Internet: www.laender-analysen.de/polen 